\title{
Global Poincaré inequalities for the composition of the sharp maximal operator and Green's operator with Orlicz norms
}

Bao Gejun ${ }^{1}$ and Yi Ling ${ }^{2 *}$

"Correspondence: yiling@desu.edu ${ }^{2}$ Department of Mathematical Science, Delaware State University, Dover, 19901, USA

Full list of author information is available at the end of the article

\begin{abstract}
In this paper, we establish the global Poincaré-type inequalities for the composition of the sharp maximal operator and Green's operator with Orlicz norm.
\end{abstract}

Keywords: Poincaré-type inequalities; Orlicz norm; sharp maximal operator; Green's operator

\section{Introduction}

The $L^{p}$-theory of solutions of the homogeneous $A$-harmonic equation $d^{\star} A(x, d u)=0$ for differential forms $u$ has been very well developed in recent years. Many $L^{p}$-norm estimates and inequalities, including the Poincaré inequalities, for solution of the homogeneous $A$-harmonic equation have been established; see $[1,2]$. The Poincaré inequalities for differential forms is an important tool in analysis and related fields, including partial differential equations and potential theory. However, the study of the nonhomogeneous $A$-harmonic equations $d^{\star} A(x, d u)=B(x, d u)$ has just begun [2-4]. In this paper, we focus on a class of differential forms satisfying the well-known nonhomogeneous $A$-harmonic equation $d^{\star} A(x, d u)=B(x, d u)$.

Let us first introduce some necessary notation and terminology. $\Omega$ will refer to a bounded, convex domain in $\mathbb{R}^{n}$ unless otherwise stated and $B$ is a ball in $\mathbb{R}^{n}, n \geq 2$. We use $\sigma B$ to denote the ball with the same center as $B$ and with $\operatorname{diam}(\sigma B)=\sigma \operatorname{diam}(B), \sigma>0$. We do not distinguish the balls from cubes in this paper. We use $|E|$ to denote the $n$ dimensional Lebesgue measure of the set $E \subseteq \mathbb{R}^{n}$. We say $w$ is a weight if $w \in L_{\text {loc }}^{1}\left(\mathbb{R}^{n}\right)$ and $w>0$ a.e. For a function $u$, we denote the average of $u$ over $B$ by

$$
u_{B}=\frac{1}{|B|} \int_{B} u d x
$$

where $|B|$ is the volume of $B$ and the $\mu$-average of $u$ over $B$ by

$$
u_{B, \mu}=\frac{1}{\mu(B)} \int_{B} u d \mu \text {. }
$$

Let $\wedge^{l}=\wedge^{l}\left(\mathbb{R}^{n}\right)$ be the set of all $l$-forms in $\mathbb{R}^{n}$, let $D^{\prime}\left(\Omega, \wedge^{l}\right)$ be the space of all differential $l$-forms on $\Omega$, and let $L^{p}\left(\Omega, \wedge^{l}\right)$ be the $l$-forms $u(x)=\sum_{I} u_{I}(x) d x_{I}$ on $\Omega$ satisfying 
$\int_{\Omega}\left|u_{I}\right|^{p} d x<\infty$ for all ordered $l$-tuples $I, l=1,2, \ldots, n$. We denote the exterior derivative by $d: D^{\prime}\left(\Omega, \wedge^{l}\right) \rightarrow D^{\prime}\left(\Omega, \wedge^{l+1}\right)$ for $l=0,1, \ldots, n-1$, and define the Hodge star operator $\star: \wedge^{k} \rightarrow \wedge^{n-k}$ as follows. If $u=u_{I} d x_{I}, i_{1}<i_{2}<\cdots<i_{k}$, is a differential $k$-form, then $\star u=(-1)^{\sum(I)} u_{I} d x_{J}$, where $I=\left(i_{1}, i_{2}, \ldots, i_{k}\right), J=\{1,2, \ldots, n\}-I$, and $\sum(I)=\frac{k(k+1)}{2}+\sum_{j=1}^{k} i_{j}$.

The Hodge codifferential operator

$$
d^{\star}: D^{\prime}\left(\Omega, \wedge^{l+1}\right) \rightarrow D^{\prime}\left(\Omega, \wedge^{l}\right)
$$

is given by $d^{\star}=(-1)^{n l+1} \star d \star$ on $D^{\prime}\left(\Omega, \wedge^{l+1}\right), l=0,1, \ldots, n-1$. We write

$$
\|u\|_{s, \Omega}=\left(\int_{\Omega}|u|^{s} d x\right)^{1 / s}
$$

The well-known nonhomogeneous $A$-harmonic equation is

$$
d^{\star} A(x, d u)=B(x, d u)
$$

where $A: \Omega \times \wedge^{l}\left(\mathbb{R}^{n}\right) \rightarrow \wedge^{l}\left(\mathbb{R}^{n}\right)$ and $B: \Omega \times \wedge^{l}\left(\mathbb{R}^{n}\right) \rightarrow \wedge^{l-1}\left(\mathbb{R}^{n}\right)$ satisfy the conditions:

$$
|A(x, \xi)| \leq a|\xi|^{p-1}, \quad A(x, \xi) \cdot \xi \geq|\xi|^{p}, \quad|B(x, \xi)| \leq b|\xi|^{p-1}
$$

for almost every $x \in \Omega$ and all $\xi \in \wedge^{l}\left(\mathbb{R}^{n}\right)$. Here, $a, b>0$ are constants and $1<p<\infty$ is a fixed exponent associated with (1). If the operator $B=0$, equation (1) becomes $d^{\star} A(x, d u)=0$, which is called the (homogeneous) $A$-harmonic equation. A solution to (1) is an element of the Sobolev space $W_{\text {loc }}^{1, p}\left(\Omega, \wedge^{l-1}\right)$ such that $\int_{\Omega} A(x, d u) \cdot d \varphi+B(x, d u) \cdot \varphi=0$ for all $\varphi \in W_{\text {loc }}^{1, p}\left(\Omega, \wedge^{l-1}\right)$ with compact support. Let $A: \Omega \times \wedge^{l}\left(\mathbb{R}^{n}\right) \rightarrow \wedge^{l}\left(\mathbb{R}^{n}\right)$ be defined by $A(x, \xi)=\xi|\xi|^{p-2}$ with $p>1$. Then $A$ satisfies the required conditions and $d^{\star} A(x, d u)=0$ becomes the $p$-harmonic equation

$$
d^{\star}\left(d u|d u|^{p-2}\right)=0
$$

for differential forms. If $u$ is a function ( 0 -form), equation (3) reduces to the usual $p$ harmonic equation $\operatorname{div}\left(\nabla u|\nabla u|^{p-2}\right)=0$ for functions. A remarkable progress has been made recently in the study of different versions of the harmonic equations, see [1] for more details.

Let $C^{\infty}\left(\Omega, \wedge^{l}\right)$ be the space of smooth $l$-forms on $\Omega$ and

$$
\mathcal{W}\left(\Omega, \wedge^{l}\right)=\left\{u \in L_{\text {loc }}^{1}\left(\Omega, \wedge^{l}\right): u \text { has generalized gradient }\right\}
$$

The harmonic $l$-fields are defined by

$$
\mathcal{H}\left(\Omega, \wedge^{l}\right)=\left\{u \in \mathcal{W}\left(\Omega, \wedge^{l}\right): d u=d^{\star} u=0, u \in L^{p} \text { for some } 1<p<\infty\right\}
$$

The orthogonal complement of $\mathcal{H}$ in $L^{1}$ is defined by

$$
\mathcal{H}^{\perp}=\left\{u \in L^{1}:\langle u, h\rangle=0 \text { for all } h \in \mathcal{H}\right\} .
$$


Then Green's operator $G$ is defined as

$$
G: C^{\infty}\left(\Omega, \wedge^{l}\right) \rightarrow \mathcal{H}^{\perp} \cap C^{\infty}\left(\Omega, \wedge^{l}\right)
$$

by assigning $G(u)$ to be the unique element of $\mathcal{H}^{\perp} \cap C^{\infty}\left(\Omega, \wedge^{l}\right)$ satisfying Poisson's equation $\Delta G(u)=u-H(u)$, where $H$ is the harmonic projection operator that maps $C^{\infty}\left(\Omega, \wedge^{l}\right)$ onto $\mathcal{H}$ so that $H(u)$ is the harmonic part of $u$. See [5] for more properties of these operators.

In harmonic analysis, a fundamental operator is the Hardy-Littlewood maximal operator. The maximal function is a classical tool in harmonic analysis but recently it has been successfully used in studying Sobolev functions and partial differential equations. For any locally $L^{s}$-integrable form $u(y)$, we define the Hardy-Littlewood maximal operator $\mathcal{M}_{s}$ by

$$
\mathcal{M}_{s}(u)=\mathcal{M}_{s}(u)(x)=\sup _{r>0}\left(\frac{1}{|B(x, r)|} \int_{B(x, r)}|u(y)|^{s} d y\right)^{\frac{1}{s}},
$$

where $B(x, r)$ is the ball of radius $r$, centered at $x, 1 \leq s<\infty$. We write $\mathcal{M}(u)=\mathcal{M}_{1}(u)$ if $s=1$. Similarly, for a locally $L^{s}$-integrable form $u(y)$, we define the sharp maximal operator $\mathcal{M}_{s}^{\#}$ by

$$
\mathcal{M}_{s}^{\#}(u)=\mathcal{M}_{s}^{\#}(u)(x)=\sup _{r>0}\left(\frac{1}{|B(x, r)|} \int_{B(x, r)}\left|u(y)-u_{B(x, r)}\right|^{s} d y\right)^{\frac{1}{s}} .
$$

Some interesting results about these operators have been established, see $[3,4]$ and $[6]$ for more details.

The purpose of this paper is to estimate the global Poincaré-type inequalities for the composition of the sharp maximal operator and Green's operator with Orlicz norm.

\section{Definitions and lemmas}

We now introduce the following definition and lemmas that will be used in this paper.

Definition 1 We say the weight $w(x)$ satisfies the $A_{r}(\Omega)$ condition, $r>1$, write $w \in A_{r}(\Omega)$ if $w(x)>1$ a.e., and

$$
\sup _{B}\left(\frac{1}{|B|} \int_{B} w d x\right)\left(\frac{1}{|B|} \int_{B}\left(\frac{1}{w}\right)^{\frac{1}{r-1}} d x\right)^{r-1}<\infty
$$

for any ball $B \subset \Omega$.

Definition 2 A proper subdomain $\Omega \subset \mathbb{R}^{n}$ is called a $\delta$-John domain, $\delta>0$, if there exists a point $x_{0} \in \Omega$ which can be joined with any other point $x \in \Omega$ by a continuous curve $\gamma \subset \Omega$ so that

$$
d(\xi, \partial \Omega) \geq \delta|x-\xi|
$$

for each $\xi \in \gamma$. Here $d(\xi, \partial \Omega)$ is the Euclidean distance between $\xi$ and $\partial \Omega$. 
Lemma 1 [7] Each $\Omega$ has a modified Whitney cover of cubes $\mathcal{V}=\left\{Q_{i}\right\}$ such that

$$
\bigcup_{i} Q_{i}=\Omega, \quad \sum_{Q_{i} \in \mathcal{V}} \chi_{\sqrt{\frac{5}{4}} Q_{i}} \leq N \chi_{\Omega}
$$

and some $N>1$, and if $Q_{i} \cap Q_{j} \neq \emptyset$, then there exists a cube $R$ (this cube need not be a member of $\mathcal{V}$ ) in $Q_{i} \cap Q_{j}$ such that $Q_{i} \cup Q_{j} \subset N R$. Moreover, if $\Omega$ is $\delta$-John, then there is a distinguished cube $Q_{0} \in \mathcal{V}$ which can be connected with every cube $Q \in \mathcal{V}$ by a chain of cubes $Q_{0}=Q_{j_{0}}, Q_{j_{1}}, \ldots, Q_{j_{k}}=Q$ from $\mathcal{V}$ and such that $Q \subset \rho Q_{j_{i}}, i=0,1,2, \ldots, k$, for some $\rho=\rho(n, \delta)$.

\section{Poincaré inequalities}

In this section, we prove the global Poincaré inequalities for the composition of the sharp maximal operator and Green's operator with $L^{p}$ norm.

To get our result, we rewrite our Theorem 2 in [4] as follows.

Lemma 2 Let $u$ be a smooth differential form satisfying A-harmonic equation (1) in a bounded domain $\Omega$, let $G$ be Green's operator, and let $\mathcal{M}_{s}^{\sharp}$ be the sharp maximal operator defined in (4) with $1<s \leq p, q<\infty$. Then there exists a constant $C$, independent of $u$, such that

$$
\begin{aligned}
& \left(\int_{B}\left|\mathcal{M}_{s}^{\sharp}(G(u))-\mathcal{M}_{s}^{\sharp}(G(u))_{B}\right|^{q} d \mu\right)^{1 / q} \\
& \quad \leq C(\delta, \Omega)|B|^{1+\frac{1}{n}-\frac{1}{p}+\frac{1}{q}}\left(\int_{\sigma B}|u|^{p} d \mu\right)^{1 / p}
\end{aligned}
$$

for all balls $B$ with $\sigma B \subset \Omega$, and a constant $\sigma>1$, where the measure $\mu$ is defined by $d \mu=$ $w(x) d x$ and $w(x) \in A_{r}(\Omega)$ with $w \geq \delta>0$ for some $r>1$ and $a$ constant $\delta$.

Theorem 1 Let $u \in L_{\mathrm{loc}}^{t}\left(\Omega, \wedge^{l}\right), l=1,2, \ldots, n$, be a smooth differential form satisfying $A$ harmonic equation (1), let $G$ be Green's operator, and let $\mathcal{M}_{s}^{\sharp}$ be the sharp maximal operator defined in (4) with $1<s<t<\infty$. Then there exists a constant $C\left(n, t, \delta_{0}, N, \Omega\right)$, independent of $u$, such that

$$
\begin{aligned}
& \left(\int_{\Omega}\left|\mathcal{M}_{s}^{\sharp}(G(u))-\left(\mathcal{M}_{s}^{\sharp}(G(u))\right)_{Q_{0}}\right|^{t} d \mu\right)^{1 / t} \\
& \quad \leq C\left(n, t, \delta_{0}, N, \Omega\right)\left(\int_{\Omega}|u|^{t} d \mu\right)^{1 / t}
\end{aligned}
$$

for any bounded and convex $\delta$-John domain $\Omega \subset \mathbb{R}^{n}$, where the fixed cube $Q_{0} \subset \Omega$, the constant $N>1$ appeared in Lemma 1 , and the measure $\mu$ is defined by $d \mu=w(x) d x$ and $w(x) \in A_{r}(\Omega)$ with $w \geq \delta_{0}>0$ for some $r>1$ and a constant $\delta_{0}$.

Proof First, we use Lemma 1 for the bounded and convex $\delta$-John domain $\Omega$. There is a modified Whitney cover of cubes $\mathcal{V}=\left\{Q_{i}\right\}$ for $\Omega$ such that $\Omega=\bigcup Q_{i}$, and $\sum_{Q_{i} \in \mathcal{V}} \chi_{\sqrt{\frac{5}{4}} Q_{i}} \leq$ $N \chi_{\Omega}$ for some $N>1$. Moreover, there is a distinguished cube $Q_{0} \in \mathcal{V}$ which can be connected with every cube $Q \in \mathcal{V}$ by a chain of cubes $Q_{0}=Q_{j_{0}}, Q_{j_{1}}, \ldots, Q_{j_{k}}=Q$ from $\mathcal{V}$ and 
such that $Q \subset \rho Q_{j i}, i=0,1,2, \ldots, k$, for some $\rho=\rho(n, \delta)$. Then, by the elementary inequality $(a+b)^{t} \leq 2^{t}\left(|a|^{t}+|b|^{t}\right), t \geq 0$, we have

$$
\begin{aligned}
& \left(\int_{\Omega}\left|\mathcal{M}_{s}^{\sharp}(G(u))-\left(\mathcal{M}_{s}^{\sharp}(G(u))\right)_{Q_{0}}\right|^{t} d \mu\right)^{1 / t} \\
& \leq\left(\sum _ { Q _ { i } \in \mathcal { V } } \left(2^{t} \int_{Q_{i}}\left|\mathcal{M}_{s}^{\sharp}(G(u))-\left(\mathcal{M}_{s}^{\sharp}(G(u))\right)_{Q_{i}}\right|^{t} d \mu\right.\right. \\
& \left.\left.\quad+2^{t} \int_{Q_{i}}\left|\left(\mathcal{M}_{s}^{\sharp}(G(u))\right)_{Q_{i}}-\left(\mathcal{M}_{s}^{\sharp}(G(u))\right)_{Q_{0}}\right|^{t} d \mu\right)\right)^{1 / t} \\
& \leq C_{1}(t)\left(\left(\sum_{Q_{i} \in \mathcal{V}} \int_{Q_{i}}\left|\mathcal{M}_{s}^{\sharp}(G(u))-\left(\mathcal{M}_{s}^{\sharp}(G(u))\right)_{Q_{i}}\right|^{t} d \mu\right)^{1 / t}\right. \\
& \left.\quad+\left(\sum_{Q_{i} \in \mathcal{V}} \int_{Q_{i}}\left|\left(\mathcal{M}_{s}^{\sharp}(G(u))\right)_{Q_{i}}-\left(\mathcal{M}_{s}^{\sharp}(G(u))\right)_{Q_{0}}\right|^{t} d \mu\right)^{1 / t}\right) .
\end{aligned}
$$

The first sum in (8) can be estimated by using Lemma 2 .

$$
\begin{aligned}
& \sum_{Q_{i} \in \mathcal{V}} \int_{Q_{i}}\left|\mathcal{M}_{s}^{\sharp}(G(u))-\left(\mathcal{M}_{s}^{\sharp}(G(u))\right)_{Q_{i}}\right|^{t} d \mu \\
& \leq C_{2}\left(n, t, \delta_{0}, \Omega\right) \sum_{Q_{i} \in \mathcal{V}} \int_{\rho_{i} Q_{i}}|u|^{t} d \mu \\
& \quad \leq C_{3}\left(n, t, \delta_{0}, \Omega\right) \sum_{Q_{i} \in \mathcal{V}} \int_{\Omega}|u|^{t} d \mu \\
& \quad \leq C_{4}\left(n, t, N, \delta_{0}, \Omega\right) \int_{\Omega}|u|^{t} d \mu,
\end{aligned}
$$

where the measure $\mu$ is defined by $d \mu=w(x) d x$ and $w(x) \in A_{r}(\Omega)$ with $w \geq \delta_{0}>0$ for some $\mathrm{r}>1$ and a constant $\delta_{0}$.

To estimate the second sum in (8), we need to use the property of $\delta$-John domain. Fix a cube $Q_{i} \in \mathcal{V}$ and let $Q_{0}=Q_{j_{0}}, Q_{j_{1}}, \ldots, Q_{j_{k}}=Q_{i}$ be the chain in Lemma 1 . Then we have

$$
\left|\left(\mathcal{M}_{s}^{\sharp}(G(u))\right)_{Q_{i}}-\left(\mathcal{M}_{s}^{\sharp}(G(u))\right)_{Q_{0}}\right| \leq \sum_{i=0}^{k-1}\left|\left(\mathcal{M}_{s}^{\sharp}(G(u))\right)_{Q_{j_{i}}}-\left(\mathcal{M}_{s}^{\sharp}(G(u))\right)_{Q_{j_{i+1}}}\right| .
$$

The chain $\left\{Q_{j_{i}}\right\}$ also has the property that for each $i, i=0,1, \ldots, k-1, Q_{j_{i}} \cap Q_{j_{i+1}} \neq \emptyset$. Thus, there exists a cube $D_{i}$ such that $D_{i} \subset Q_{j_{i}} \cap Q_{j_{i+1}}$ and $Q_{j_{i}} \cup Q_{j_{i+1}} \subset N D_{i}, N>1$. So,

$$
\frac{\max \left\{\left|Q_{j_{i}}\right|,\left|Q_{j_{i+1}}\right|\right\}}{\left|Q_{j_{i}} \cap Q_{j_{i+1}}\right|} \leq \frac{\max \left\{\left|Q_{j_{i}}\right|,\left|Q_{j_{i+1}}\right|\right\}}{\left|D_{i}\right|} \leq N
$$

Note that

$$
\mu(Q)=\int_{Q} d \mu=\int_{Q} w(x) d x \geq \int_{Q} \delta_{0} d x=\delta_{0}|Q|
$$


By (11), (12) and Lemma 2, we have

$$
\begin{aligned}
& \left|\left(\mathcal{M}_{s}^{\sharp}(G(u))\right)_{Q_{j_{i}}}-\left(\mathcal{M}_{s}^{\sharp}(G(u))\right)_{Q_{j_{i+1}}}\right|^{t} \\
& \quad=\frac{1}{\mu\left(Q_{j_{i}} \cap Q_{j_{i+1}}\right)} \int_{Q_{j_{i}} \cap Q_{j_{i+1}}}\left|\left(\mathcal{M}_{s}^{\sharp}(G(u))\right)_{Q_{j_{i}}}-\left(\mathcal{M}_{s}^{\sharp}(G(u))\right)_{Q_{j_{i+1}}}\right|^{t} d \mu \\
& \quad \leq \frac{1}{\delta_{0}\left|Q_{j_{i}} \cap Q_{j_{i+1}}\right|} \int_{Q_{j_{i}} \cap Q_{j_{i+1}}}\left|\left(\mathcal{M}_{s}^{\sharp}(G(u))\right)_{Q_{j_{i}}}-\left(\mathcal{M}_{s}^{\sharp}(G(u))\right)_{Q_{j_{i+1}}}\right|^{t} d \mu \\
& \quad \leq \frac{N}{\delta_{0} \max \left\{\left|Q_{j_{i}}\right|,\left|Q_{j_{i+1}}\right|\right\}} \int_{Q_{j_{i}} \cap Q_{j_{i+1}}}\left|\left(\mathcal{M}_{s}^{\sharp}(G(u))\right)_{Q_{j_{i}}}-\left(\mathcal{M}_{s}^{\sharp}(G(u))\right)_{Q_{j_{i+1}}}\right|^{t} d \mu \\
& \quad \leq C_{5}\left(n, t, \delta_{0}, N, \Omega\right) \sum_{k=i}^{i+1} \frac{1}{\left|Q_{j_{k}}\right|} \int_{Q_{j_{k}}}\left|\mathcal{M}_{s}^{\sharp}(G(u))-\left(\mathcal{M}_{s}^{\sharp}(G(u))\right)_{Q_{j_{k}}}\right|^{t} d \mu \\
& \quad \leq C_{6}\left(n, t, \delta_{0}, N, \Omega\right) \sum_{k=i}^{i+1} \frac{\left|Q_{j_{k}}\right|^{1+\frac{1}{n}}}{\left|Q_{j_{k}}\right|} \int_{\sigma_{j_{k}} Q_{j_{k}}}|u|^{t} d \mu \\
& \quad=C_{6}\left(n, t, \delta_{0}, N, \Omega\right) \sum_{k=i}^{i+1}\left|Q_{j_{k}}\right|^{\frac{1}{n}} \int_{\sigma_{j_{k}} Q_{j_{k}}}|u|^{t} d \mu \\
& \quad \leq C_{7}\left(n, t, \delta_{0}, N, \Omega\right) \sum_{k=i}^{i+1}|\Omega|^{\frac{1}{n}} \int_{\Omega}|u|^{t} d \mu \\
& \quad \leq C_{8}\left(n, t, \delta_{0}, N, \Omega\right) \sum_{Q_{i} \in \mathcal{V}} \int_{\Omega}|u|^{t} d \mu \\
& \quad \leq C_{9}\left(n, t, \delta_{0}, N, \Omega\right) \int_{\Omega}^{|u|^{t} d \mu .}
\end{aligned}
$$

Then, by (10), (13) and the elementary inequality $\left|\sum_{i=1}^{M} t_{i}\right|^{s} \leq M^{s-1} \sum_{i=1}^{M}\left|t_{i}\right|^{s}$, we finally obtain

$$
\begin{aligned}
& \sum_{Q_{i} \in \mathcal{V}} \int_{Q_{i}}\left|\left(\mathcal{M}_{s}^{\sharp}(G(u))\right)_{Q_{i}}-\left(\mathcal{M}_{s}^{\sharp}(G(u))\right)_{Q_{0}}\right|^{t} d \mu \\
& \quad \leq C_{10}\left(n, t, \delta_{0}, N, \Omega\right) \sum_{Q_{i} \in \mathcal{V}} \int_{Q_{i}}\left(\int_{\Omega}|u|^{t} d \mu\right) d \mu \\
& \quad=C_{10}\left(n, t, \delta_{0}, N, \Omega\right)\left(\sum_{Q_{i} \in \mathcal{V}} \int_{Q_{i}} d \mu\right) \int_{\Omega}|u|^{t} d \mu \\
& \leq C_{11}\left(n, t, \delta_{0}, N, \Omega\right)\left(\int_{\Omega} d \mu\right) \int_{\Omega}|u|^{t} d \mu \\
& \quad=C_{11}\left(n, t, \delta_{0}, N, \Omega\right) \mu(\Omega) \int_{\Omega}|u|^{t} d \mu \\
& \quad=C_{12}\left(n, t, \delta_{0}, N, \Omega\right) \int_{\Omega}|u|^{t} d \mu .
\end{aligned}
$$

Substituting (9) and (14) in (8), we have completed the proof of Theorem 1. 


\section{Poincaré inequality with Orlicz norm}

In this section, we give a global Poincaré inequality with Orlicz norm for the composition of the sharp maximal operator and Green's operator.

Definition 3 Let $\varphi$ be a continuously increasing convex function on $[0, \infty)$ with $\varphi(0)=0$, and let $\Lambda$ be a domain with $\mu(\Lambda)<\infty$. If $u$ is a measurable function in $\Lambda$, then we define the Orlicz norm of $u$ by

$$
\|u\|_{L(\varphi, \Lambda, \mu)}=\inf \left\{k>0: \frac{1}{\mu(\Lambda)} \int_{\Lambda} \varphi\left(\frac{|u(x)|}{k}\right) d \mu \leq 1\right\}
$$

A continuously increasing function $\psi:[0, \infty) \rightarrow[0, \infty)$ with $\varphi(0)=0$ is called an Orlicz function. A convex Orlicz function $\varphi$ is often called a Young function.

In [8], Buckley and Koskela gave the following class of functions.

Definition 4 We say a Young function $\varphi$ lies in the class $G(p, q, C), 1 \leq p<q<\infty, C \geq 1$, if (i) $1 / C \leq \varphi\left(t^{1 / p}\right) / g(t) \leq C$ and (ii) $1 / C \leq \varphi\left(t^{1 / q}\right) / h(t) \leq C$ for all $t>0$, where $g$ is a convex increasing function and $h$ is a concave increasing function on $[0, \infty)$.

From [8] and [9], we know that the class $G(p, q, C)$ contains some very interesting functions, such as $\varphi(t)=t^{p}$ and $\varphi(t)=t^{p} \log _{+}^{\alpha}(t), p \geq 1, \alpha \in \mathbb{R}$, and each of $\varphi, g$ and $h$ is doubling in the sense that its values at $t$ and $2 t$ are uniformly comparable for all $t>0$, and the consequent fact that

$$
C_{1} t^{q} \leq h^{-1}(\varphi(t)) \leq C_{2} t^{q}, \quad C_{1} t^{p} \leq g^{-1}(\varphi(t)) \leq C_{2} t^{p},
$$

where $C_{1}$ and $C_{2}$ are constants.

Now, we are ready to give our another global Poincaré inequality with Orlicz norm.

Theorem 2 Let $\varphi$ be a Young function in the class $G\left(p, q, C_{0}\right), 1 \leq p<q<\infty, C_{0} \geq 1$, let $u \in$ $L_{\mathrm{loc}}^{t}\left(\Omega, \wedge^{l}\right), l=1,2, \ldots, n$, be a smooth differential form satisfying A-harmonic equation (1) in $\Omega$, let $G$ be Green's operator, and let $\mathcal{M}_{s}^{\sharp}$ be the sharp maximal operator defined in (4) with $1<s \leq t<\infty$. Then there exists a constant $C$, independent of $u$, such that

$$
\left\|\mathcal{M}_{s}^{\sharp}(G(u))-\mathcal{M}_{s}^{\sharp}(G(u))_{Q_{0}}\right\|_{L(\varphi, \Omega, \mu)} \leq C\|u\|_{L(\varphi, \Omega, \mu)}
$$

for any bounded and convex $\delta$-John domain $\Omega \subset \mathbb{R}^{n}$ with $\mu(\Omega)<\infty$, where the fixed cube $Q_{0} \subset \Omega$ appeared in Lemma 1 , and the measure $\mu$ is defined by $d \mu=w(x) d x$ and $w(x) \in$ $A_{r}(\Omega)$ with $w \geq \delta_{0}>0$ for some $r>1$ and a constant $\delta_{0}$.

Proof Let $g, h$ be the functions in the $G\left(p, q, C_{0}\right)$ condition. Note that $\varphi$ is an increasing function. Using Theorem 1, (i) in Definition 4, and Jensen's inequality, we obtain

$$
\begin{aligned}
\varphi( & \left.\frac{1}{k}\left(\int_{\Omega}\left|\mathcal{M}_{s}^{\sharp}(G(u))-\mathcal{M}_{s}^{\sharp}(G(u))_{Q_{0}}\right|^{t} d \mu\right)^{1 / t}\right) \\
& \leq \varphi\left(\frac{1}{k} C_{1}\left(\int_{\Omega}|u|^{t} d \mu\right)^{1 / t}\right)
\end{aligned}
$$




$$
\begin{aligned}
& =\varphi\left(\left(\frac{1}{k^{t}} C_{1}^{t} \int_{\Omega}|u|^{t} d \mu\right)^{1 / t}\right) \\
& \leq C_{0} g\left(\frac{1}{k^{t}} C_{1}^{t} \int_{\Omega}|u|^{t} d \mu\right) \\
& =C_{0} g\left(\int_{\Omega} \frac{1}{k^{t}} C_{1}^{t}|u|^{t} d \mu\right) \\
& \leq C_{0} \int_{\Omega} g\left(\frac{1}{k^{t}} C_{1}^{t}|u|^{t}\right) d \mu .
\end{aligned}
$$

Again, from (i) in Definition 4, we have

$$
g(x) \leq C_{0} \varphi\left(x^{\frac{1}{t}}\right) .
$$

Thus, we obtain

$$
\int_{\Omega} g\left(\frac{1}{k^{t}} C_{1}^{t}|u|^{t}\right) d \mu \leq C_{0} \int_{\Omega} \varphi\left(\frac{1}{k} C_{1}|u|\right) d \mu
$$

Combining (17) and (18) yields

$$
\begin{aligned}
\varphi( & \left.\frac{1}{k}\left(\int_{\Omega}\left|\mathcal{M}_{s}^{\sharp}(G(u))-\mathcal{M}_{s}^{\sharp}(G(u))_{Q_{0}}\right|^{t} d \mu\right)^{1 / t}\right) \\
& \leq C_{0}^{2} \int_{\Omega} \varphi\left(\frac{1}{k} C_{1}|u|\right) d \mu \\
& =C_{2} \int_{\Omega} \varphi\left(\frac{1}{k} C_{1}|u|\right) d \mu .
\end{aligned}
$$

Now, using Jensen's inequality for $h^{-1},(16)$ and (ii) in Definition 4, and noticing that $\varphi$ is doubling, we see

$$
\begin{aligned}
& \int_{\Omega} \varphi\left(\frac{\left|\mathcal{M}_{s}^{\sharp}(G(u))-\mathcal{M}_{s}^{\sharp}(G(u))_{Q_{0}}\right|}{k}\right) d \mu \\
& =h\left(h^{-1}\left(\int_{\Omega} \varphi\left(\frac{\left|\mathcal{M}_{s}^{\sharp}(G(u))-\mathcal{M}_{s}^{\sharp}(G(u))_{Q_{0}}\right|}{k}\right) d \mu\right)\right) \\
& \quad \leq h\left(\int_{\Omega} h^{-1}\left(\varphi\left(\frac{\left|\mathcal{M}_{s}^{\sharp}(G(u))-\mathcal{M}_{s}^{\sharp}(G(u))_{Q_{0}}\right|}{k}\right)\right) d \mu\right) \\
& \quad \leq h\left(C_{3} \int_{\Omega}\left(\frac{\left|\mathcal{M}_{s}^{\sharp}(G(u))-\mathcal{M}_{s}^{\sharp}(G(u))_{Q_{0}}\right|}{k}\right)^{t} d \mu\right) \\
& \leq C_{0} \varphi\left(\left(C_{3} \int_{\Omega}\left(\frac{\left|\mathcal{M}_{s}^{\sharp}(G(u))-\mathcal{M}_{s}^{\sharp}(G(u))_{Q_{0}}\right|}{k}\right)^{t} d \mu\right)^{\frac{1}{t}}\right) \\
& =C_{0} \varphi\left(\frac{1}{k}\left(C_{3} \int_{\Omega}\left(\left|\mathcal{M}_{s}^{\sharp}(G(u))-\mathcal{M}_{s}^{\sharp}(G(u))_{Q_{0}}\right|\right)^{t} d \mu\right)^{\frac{1}{t}}\right) \\
& \leq C_{4} \varphi\left(\frac{1}{k}\left(\int_{\Omega}\left(\left|\mathcal{M}_{s}^{\sharp}(G(u))-\mathcal{M}_{s}^{\sharp}(G(u))_{Q_{0}}\right|\right)^{t} d \mu\right)^{\frac{1}{t}}\right) .
\end{aligned}
$$


Substituting (19) into (20) and using the fact that $\varphi$ is doubling, we get

$$
\begin{aligned}
& \int_{\Omega} \varphi\left(\frac{\left|\mathcal{M}_{s}^{\sharp}(G(u))-\mathcal{M}_{s}^{\sharp}(G(u))_{Q_{0}}\right|}{k}\right) d \mu \\
& \quad \leq C_{5} \int_{\Omega} \varphi\left(\frac{1}{k} C_{1}|u|\right) d \mu \\
& \quad \leq C_{6} \int_{\Omega} \varphi\left(\frac{1}{k}|u|\right) d \mu .
\end{aligned}
$$

Therefore, from Definition 3, we have

$$
\left\|\mathcal{M}_{s}^{\sharp}(G(u))-\mathcal{M}_{s}^{\sharp}(G(u))_{Q_{0}}\right\|_{L(\varphi, \Omega, \mu)} \leq C_{6}\|u\|_{L(\varphi, \Omega, \mu)} .
$$

\section{Competing interests}

The authors declare that they have no competing interests.

\section{Authors' contributions}

All authors completed the paper together. All authors read and approved the final manuscript.

\section{Author details}

${ }^{1}$ Department of Mathematics, Harbin Institute of Technology, Harbin, 150001, P.R. China. ${ }^{2}$ Department of Mathematical Science, Delaware State University, Dover, 19901, USA.

\section{Acknowledgements}

The first author was supported by NSF of P.R. China (No. 11071048).

Received: 23 April 2013 Accepted: 8 October 2013 Published: 11 Nov 2013

\section{References}

1. Agarwal, RP, Ding, S, Nolder, CA: Inequalities for Differential Forms. Springer, Berlin (2009)

2. Agarwal, RP, Ding, S: Global Caccioppoli-type and Poincaré inequalities with Orlicz norms. J. Inequal. Appl. 2010, Article ID 727954 (2010)

3. Ling, Y, Umoh, HM: Global estimates for singular integrals of the composition of the maximal operator and the Green's operator. J. Inequal. Appl. 2010, Article ID 723234 (2010)

4. Ling, Y, Gejun, B: Some local Poincaré inequalities for the composition of the sharp maximal operator and the Green's operator. Comput. Math. Appl. 63, 720-727 (2012)

5. Warner, FW: Foundations of Differentiable Manifolds and Lie Groups. Springer, New York (1983)

6. Ding, S: Norm estimate for the maximal operator and Green's operator. Dyn. Contin. Discrete Impuls. Syst., Ser. A Math. Anal. 16, 72-78 (2009). Differential Equations and Dynamical Systems, suppl. S1

7. Nolder, CA: Hardy-Littlewood theorems for A-harmonic tensors. III. J. Math. 43, 613-631 (1999)

8. Buckley, SM, Koskela, P: Orlicz-Hardy inequalities. III. J. Math. 48, 787-802 (2004)

9. Ding, S: $L(\varphi, \mu)$-averaging domains and Poincaré inequalities with Orlicz norm. Nonlinear Anal. 73, 256-265 (2010) 